\title{
ENTROPY GENERATION DUE TO NATURAL CONVECTION WITH NON -UNIFORM HEATING OF POROUS QUADRANTAL ENCLOSURE-A NUMERICAL STUDY
}

\author{
Shantanu Dutta*, Arup Kumar Biswas \\ Department of Mechanical Engineering, National Institute of Technology, Durgapur-713209, INDIA
}

\begin{abstract}
Industrial processes optimization for higher energy efficiency may be effectively carried out based on the thermodynamic approach of entropy generation minimization (EGM). This approach provides the key insights on how the available energy (exergy) is being destroyed during the process and the ways to minimize its destruction. In this study, EGM approach is implemented for the analysis of optimal thermal mixing and temperature uniformity due to natural convection in quadrantal cavity filled with porous medium for the material processing applications or for cooling of electrical equipments. Effect of the permeability of the porous medium and the role of non-uniform heating in enhancing the thermal mixing, temperature effects and minimization of entropy generation is analyzed. The numerical solutions are obtained using finite element method. Stream lines, Isotherms and Entropy generation results are depicted for $\mathrm{Ra}=10^{6}, 1.7 \times 10^{5}, 10^{4}$ and for $\mathrm{Da}=10^{-3}, 10^{-4}$ and $10^{-5}$ at $\mathrm{Pr}=0.71$.It is found that at lower Darcy number (Da), the thermal mixing is low and the heat transfer irreversibility dominates the total entropy generation. In contrast, thermal mixing is improved due to enhanced convection at higher $\mathrm{Da}$. It is observed that fluid friction irreversibility dominates over heat transfer irreversibility for only $\mathrm{Da}=10^{-3}$ at $\mathrm{Ra}=10^{6}$. The local entropy generation is maximum at the bottom wall, while at the center and top of the enclosure it becomes minimum. Based on EGM analysis, it is established that total entropy production is not significant with larger thermal mixing at high Darcy number, and can be recommended for material processing process or for cooling of electrical equipments.
\end{abstract}

Keywords: Natural convection; Quadrantal enclosure; Non- linear heating; Bejan number.

\section{INTRODUCTION}

Natural convection phenomenon is a very interesting topic for five decades since it plays a vital role in many engineering applications. Some of the recent investigations on natural convection in fluid saturated porous enclosures particularly focus on flow and heat transfer,

but not effectively on energy distribution. There are many published studies which are related to natural convection in rectangular porous enclosures in the past. Moya et al.(1987), Bejan (1979), Prasad and Kulacki (1984), Baytas and Pop (1999), Baytas (1999) have published many important results for this types of enclosure.

Waheed (2009) studied the problem of the natural laminar convection in square enclosures filled with fluid-saturated porous medium numerically in his work using the heat-function formulation approach. The flow governing equations including the Brinkmanextended Darcy equations of motion, energy, and heat-function equations were thereby solved using the finite-difference method. The results showed that all the problem governing parameters have strong effect on the convection vigour, isotherms, and heat-function fields and profiles. They also found that an increase in the value of the Darcy number above unity had no more influence on the heat-function profiles. They also gave a clear picture of the heat trajectory in the enclosure which became possible through the use of the heat lines. The heat lines approach thus made possible the interpretation of the flow and the temperature fields, and thus provided a better means to explain the convective heat transfer in the cavity filled with fluid-saturated porous media. Moukalled and Darwish (2010) presented numerical solutions for laminar natural convection heat transfer in a fluid saturated porous enclosure between two isothermal concentric cylinders of rhombic cross sections. They found that the flow strength and convection heat transfer increase with an increase in $\mathrm{Ra}, \mathrm{Da}, \mathrm{E}_{\mathrm{g}}$ (enclosure gap ratio), and/or $\varepsilon$ (porosity). At low $\mathrm{E}_{\mathrm{g}}$ values, the flow in the enclosure observed was weak and convection heat transfer was low even though the total heat transfer was found to be higher at higher $\mathrm{E}_{\mathrm{g}}$ values, due to an increase in conduction heat transfer. An increase in Pr was associated with a decrease in the flow strength and an increase in total heat transfer. They also stated that convection started affecting the total heat transfer at Ra values higher than the critical one. The critical Ra decreased with increasing Da and/or $\varepsilon$, and increased with decreasing $\mathrm{E}_{\mathrm{g}}$ in that study. Another interesting problem was reported by Wong and Xie (2011) who studied the classical/spectral conjugate gradient methods with adjoint equations which were applicable to the natural convection problem in a porous medium for the determination of an unknown heat source. The direct, sensitivity and adjoint equations associated with the Darcy and the Forchheimer terms were given for a Boussinesq fluid, over a square porous medium in two dimensions. The inverse solutions that were obtained was determined by a second-order scheme in space and in time and further a mixed finite element method were presented for a square enclosure under known temperature boundary conditions. Questions regarding the numerical accuracy of the proposed schemes for porous flow models for recovering the strength of the unknown heat source were also addressed in that research article. Also Bhuvaneswari et al.(2011) discussed about convective flow and heat transfer in a cavity in the presence of uniform magnetic field and discussed about different combinations of phase deviation, amplitude ratio, and Hartmann and Rayleigh numbers. It was observed by them that the heat transfer rate increased with amplitude ratio. They also stated that the heat transfer rate first increased and then decreased on increasing the

\footnotetext{
*Corresponding Author. Email: shantanudut@gmail.com.
} 
phase deviation. They also found that the heat transfer rate decreased with an increasing Hartmann number. Pandit and Chattopadhyay (2014) employed higher order compact scheme to investigate the transient natural convection in a deep enclosure filled with porous medium. Also Chou et al.(2015) analyzed and employed the effects of temperaturedependent viscosity on natural convection inside porous media between two concentric spheres.

Gibanov et al (2017) carried out numerical analysis of natural convection combined with entropy for a ferrofluid under the effect of inclined uniform magnetic field. They used the governing equations with corresponding boundary conditions formulated in dimensionless stream function and vorticity using Brinkman-extended Darcy model for porous layer and further they were solved numerically using finite difference method. The published results by them showed that an inclusion of spherical ferric oxide nano-particles could lead to a diminution of entropy generation in the case of similar flow and heat transfer structures. Entropy generation and heat flow analysis study on nanoparticle added fluids, namely nano fluids, are increasing rapidly in recent years in different shaped cavities or nano and micro channels. A numerical analysis of laminar natural convection with entropy generation in a partially heated open triangular cavity filled with a $\mathrm{Cu}$-water nano-fluid was carried out by Bondareva et al.(2016). The main findings of that paper was that there was heat transfer enhancement and fluid flow attenuation with nanoparticles volume fraction, mainly for high values of Rayleigh number. Sheremet et.al(2017) also carried out a numerical study on entropy generation in natural convection of nanofluid in a wavy shaped cavity using a single-phase nanofluid model. They also found that the average Bejan number is an increasing function of nanoparticle volume fraction and a decreasing function of the Rayleigh number, undulation number and wavy contraction ratio. Also, they investigated and found that an increase in the wavy contraction ratio leads to an attenuation of the convective flow due to an intensification of the secondary vortices located in the bottom and upper wavy troughs. It was also found by them that the solid volume fraction suppresses the fluid motion. At the same time, it was also found that an increase in the nanoparticle volume fraction leads to an enhancement of the heat transfer rate and the average Bejan number while the average entropy generation decreased.

The work on quadrantal cavity has been done earlier by Aydin and Yesiloz (2011) and Yesiloz and Aydin (2011).They investigated experimentally and numerically the effects of the inclination angle $(\varphi)$, and the Rayleigh number, Ra on fluid flow and heat transfer for the range of angle of inclination between $0^{0} \leq \varphi \leq 360^{\circ}$, and Ra from $10^{5}$ to $10^{7}$. It was disclosed by them that heat transfer changes dramatically according to the inclination angle which affects convection currents inside, i.e. flow physics inside. Sen et al.(2013,2015) performed numerical investigation to analyze the natural convection heat transfer in quadrantal cavity having hot bottom wall and cold curved wall and using heater on adjacent walls for Rayleigh number in the range of $10^{3} \leq \mathrm{Ra} \leq 10^{7}$ and found out that both flow and temperature fields are affected by a changing Ra. They also found out that heat transfer increases with increase in Rayleigh number and the flow strength increases with increase in size of heater on the vertical wall compared to the bottom wall and temperature fields were also affected. In contrast, with increase in size of heater on both side of adjacent walls flow strength did not change significantly. Bose et al. (2013) also performed numerical simulation to study the natural convection heat transfer in quadrantal cavity having finned hot vertical wall and cold bottom wall for Rayleigh number in the range of $10^{4} \leq \mathrm{Ra}$ $\leq 10^{6}$. They found out that heat transfer increases with increase of Rayleigh number and they also found out that the values of stream function (flow strength) also reportedly increase with the increasing of Rayleigh number. They also found out that non-dimensional fin location changes the shape of vortices and enhances the strength of the flow and with the increasing of dimensionless fin length, the fin causes blockage effect for the flow. Very recently Dutta et al.(2018) have numerically investigated porous quadrantal enclosure with sinusoidal heating of bottom wall and cold vertical wall and insulated curved wall and found the effect of Darcy number is significant in dictating the Nusselt number only for higher values of Rayleigh number and the variation is more profound for larger values of Darcy number. The variation of entropy generation rate was found to be significant with the Darcy number only for higher values of Rayleigh number.

We also know that the available energy supply or energy conservation practices truly balance the energy efficiency, while heat transfer enhancement is used in combination with the effective energy distribution. Hence, the principles of fluid mechanics and heat transfer go hand in hand with the second law of thermodynamics to determine energy efficiency and equals to or approaches sustainable energy improvements of the system and processes. This approach of thermodynamic optimization known as entropy generation minimization (EGM) was first reported by Bejan (1996) to optimize any process or system by minimizing the irreversibilities present in the system. Varol et al. (2009) analyzed the entropy generation during combined convection and conduction inside the right angle trapezoidal enclosure filled with fluid saturated porous medium via considering major controlling factors, such as thermal conductivity ratio and dimensionless thickness of the solid wall on heat transfer and fluid flow. Results of entropy generation analysis for natural convection inside a porous square enclosure was presented by Zahmatkesh (2008) in terms of streamlines, isothermal lines, iso-entropy generation lines, and iso-Bejan lines for various thermal boundary conditions, and it was concluded by them that thermal boundary conditions greatly influence the average Nusselt number, global entropy generation rate, and global Bejan number in the calculated range of Darcy-modified Rayleigh number.

The objective of this present study is to analyze the entropy generation due to natural convection in quadrantal enclosure filled with fluid saturated porous medium for Rayleigh Benard heating situations based on its various engineering (cooling of mounted electronic devices) and natural applications(geothermal).The finite element method has been employed to solve the nonlinear equations of fluid flow, energy and entropy for a range of Darcy parameters $\mathrm{Da}=10^{-5}-10^{-3}$ and $\mathrm{Pr}=0.71$ for Rayleigh number $\left(\mathrm{Ra}=10^{4}, 1.7 \times 10^{5}\right.$ and $\left.10^{6}\right)$. Also important to emphasise here is that the numerical simulations are chosen in order to show the effect of Da which are reported to be well within the regimes of possible operating conditions as reported in the literature by Basak et al.( 2012,2013). It is also important to mention in this context here that the Darcy number physically represents the permeability of the porous medium chosen for the numerical study, while the Rayleigh number represents the relative ability to transport thermal energy due to buoyancy to that due to diffusion of heat in the enclosure or cavity chosen for study. The results are presented in terms of contours of isotherms $(\theta)$, streamlines $(\psi)$, entropy generation maps due to heat transfer $\left(\mathrm{S}_{\theta}\right)$, and entropy generation maps due to fluid friction $\left(S_{\psi}\right)$. In many industrial or practical situations we frequently encounter with horizontal cylinders filled with fluids. This type of geometry and flow configuration discussed in the present article have relevance to those cases discussed above and are also commonly observed in the field of electronics, cooling system, heat exchanger, etc. Also, as per authors knowledge very less work regarding natural convection in quadrantal enclosures with porous media with sinusoidal heated bottom wall and neighbouring curved and vertical walls have been reported earlier, which lead to motivation of this numerical study. As to the best of my knowledge, this is the first natural convection study in quadrantal cavity with non-linear heating of hot bottom wall filled with porous material with the following boundary conditions.

\section{PROBLEM DESCRIPTION}

The quadrantal enclosure chosen for our study is filled with porous material inside and is represented in Figure 1a. The grid (Figure 1b) build by free triangular method is also shown to the right. The bottom wall is 
the hot-wall imposed with non uniform heating and the vertical wall and the curved wall is maintained at lower temperature than the hot bottom wall of the enclosure and is defined as the cold-walls. Thermo physical properties of the fluid in the flow field are assumed to be constant, except the density in buoyancy term, and change in density due to temperature variation is calculated using Boussinesq's approximation. It may be noted that the local thermal equilibrium (LTE) is valid, i.e., the temperature of the fluid phase $\left(\mathrm{T}_{\mathrm{f}}\right)$ is equal to the temperature of the solid phase $\left(\mathrm{T}_{\mathrm{s}}\right)$ within the porous medium, and similar approximations were also used by earlier researchers(refer Nield and Bejan(2006)). The momentum transport in porous medium is based on a generalized non-Darcy model. However, the velocity square term or Forchheimer term which models the inertia effect is neglected here in the present case, as this work deals with natural convection flow within a porous enclosed cavity. However, the current model involves an advection term, as well as Brinkman terms, to incorporate non-Darcy effects(refer Vafai and Tien (1981)).Under these assumptions, the governing equations for steady laminar natural convection flow in a porous rhombic cavity for conservation of mass, momentum, and energy equations may be written with the following dimensionless variables or numbers.
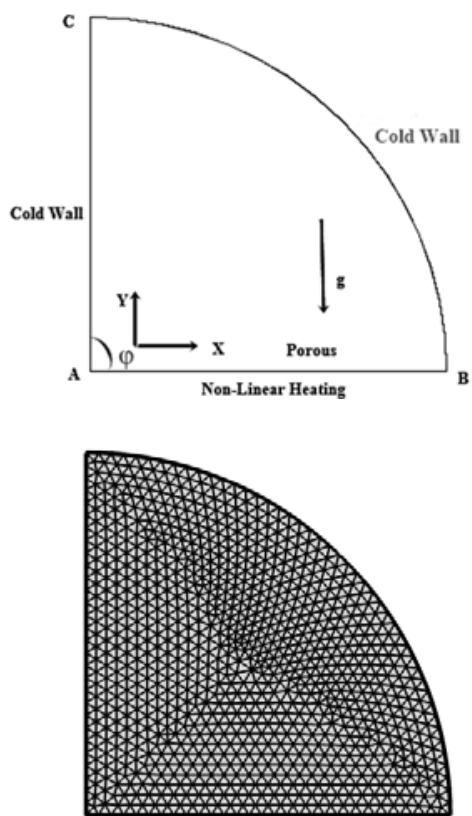

Fig. 1(a) Geometry of the problem, and (b) Grid distribution

\subsection{Boundary Conditions}

For the enclosure of Figures1(a) the boundary conditions and no slip condition is imposed on all walls. The curved and vertical wall of quadrantal enclosure are maintained at the cold temperature and bottom wall is imposed with a non-uniform varying temperature distribution. The boundary conditions are:

$U=V=0, \theta=0$ (along BC)

The dimensional form of the non uniform temperature distribution on the heated wall is given as per Sarris et al.(2002)

$T_{w}^{*}\left(x^{*}\right)=T_{C}^{*}+\frac{\Delta T^{*}}{2}\left(1-\cos \left(\frac{2 \pi x^{*}}{L}\right)\right)$

where $\Delta T^{*}$ is the temperature difference between the maximum and minimum temperatures of the heated wall, $T_{c}{ }^{*}$ is the temperatures at the cold wall, and $L$ is the length of the cavity. The dimensionless form of the temperature distribution on the heated bottom wall (Eq. (3)) can be written by using scale parameters given by Dalal and Das (2005) as
$T_{w}(x)=\frac{1}{2}(1-\cos (2 \pi x)$ (along $\mathrm{AB})$

Also, $U=V=0, \quad$ (also, along $\mathrm{AB})$

On the vertical wall (along AC)

$\theta=0, U=V=0$ and $0<X<1 ; 0<\mathrm{Y}<1$

\subsection{Governing Equations}

The momentum transport in porous medium is based on a generalized non-Darcy model as already discussed. However, the velocity square term or Forchheimer term which models the inertia effect is neglected here in the present case, as this work deals with natural convection flow within a porous enclosed cavity.

The major assumptions made to analyze the present problem are as follows:

(i) The fluid confined within porous bed is Newtonian and the flow is steady, laminar and incompressible.

(ii) The effect of viscous dissipation is neglected.

(iii) The physical properties except the density in the body force term are considered to be constant. The variation of density in the body force term with temperature follows Boussinesq approximation.

(iv) The radiation heat transfer is neglected.

(v) Darcy-Forchheimer model is used to simulate the momentum transfer in the porous medium.

(vi) The temperature of the fluid phase is equal to the temperature of the solid phase everywhere and local thermodynamic-equilibrium is applicable.

Under these assumptions, the governing equations for steady two-dimensional natural convection flow in a porous rhombic cavity for conservation of mass, momentum, and energy may be written with the following dimensionless variables. $\mathrm{H}=$ height of Enclosure, $\mathrm{L}=$ Length of Enclosure and where Pr is Prandtl number and Ra is Rayleigh number and Da is Darcy number.

$$
\begin{aligned}
& \frac{\partial U}{\partial X}+\frac{\partial V}{\partial Y}=0 \\
& U \frac{\partial U}{\partial X}+V \frac{\partial U}{\partial Y}=-\frac{\partial P}{\partial X}-\operatorname{Pr} \cdot \frac{U}{D a}+\operatorname{Pr}\left(\frac{\partial^{2} U}{\partial X^{2}}+\frac{\partial^{2} U}{\partial Y^{2}}\right) \\
& U \frac{\partial V}{\partial X}+V \frac{\partial V}{\partial Y}=-\frac{\partial P}{\partial Y}-\operatorname{Pr} \cdot \frac{V}{D a}+\operatorname{Pr}\left(\frac{\partial^{2} V}{\partial X^{2}}+\frac{\partial^{2} V}{\partial Y^{2}}\right)+R a * \operatorname{Pr}^{*} \theta
\end{aligned}
$$

$$
U \frac{\partial \theta}{\partial X}+V \frac{\partial \theta}{\partial Y}=\frac{\partial^{2} \theta}{\partial X^{2}}+\frac{\partial^{2} \theta}{\partial Y^{2}}
$$

In the above equations, various dimensionless parameters are defined as

$$
\begin{aligned}
& \theta=\frac{T-T_{c}}{T_{h}-T_{c}}, p=\frac{p L^{2}}{\rho \alpha^{2}}, \operatorname{Pr}=\frac{v}{\alpha}, \\
& R a=\frac{g \beta\left(T_{h}-T_{c}\right) L^{3} \operatorname{Pr}}{v^{2}} /=\frac{c_{p} \rho^{2} g \beta T L^{3}}{\mu k} \\
& X=\frac{x}{L} ; Y=\frac{y}{L}, U=\frac{u L}{\alpha}, V=\frac{v L}{\alpha}, D a=\frac{K}{L^{2}}
\end{aligned}
$$

where $\mathrm{u}$ and $\mathrm{v}$ are the dimensional velocities in $\mathrm{x}$ and $\mathrm{y}$ directions, respectively, $\alpha$ is the thermal diffusivity, $\mathrm{p}$ is the dimensional pressure, $\rho$ is the density of fluid, $\mathrm{g}$ is gravitational acceleration, $\beta$ is volumetric thermal expansion coefficient, $v$ is kinematic viscosity, $\mathrm{K}$ is the permeability of the porous media. 


\subsection{Stream Function}

The fluid motion is displayed using the stream function $(\psi)$ obtained from velocity components ( $\mathrm{U}$ and $\mathrm{V})$. The relationships between stream function and velocity components yield a single equation which is depicted as:

$\frac{\partial^{2} \psi}{\partial X^{2}}+\frac{\partial^{2} \psi}{\partial Y^{2}}=\frac{\partial U}{\partial Y}-\frac{\partial V}{\partial X}$

The no-slip condition is valid at all boundaries and there is no cross flow, hence $\psi=0$ is used in residual equations at the nodes for the boundaries. Using the above definition of the stream function, the positive sign of $\psi$ denotes anti-clockwise circulation and the clockwise circulation is represented by the negative sign of $\psi$.

\subsection{Entropy Generation}

According to local thermodynamic equilibrium of linear transport theory, the dimensionless total local entropy generation for a two-dimensional heat and fluid flow in porous applications in cartesian coordinates in explicit form is written as:

$S_{\theta}=\left(\frac{\partial \theta}{\partial X}\right)^{2}+\left(\frac{\partial \theta}{\partial Y}\right)^{2}$

$s_{\psi}=\xi *\left[\left(U^{2}+V^{2}\right)+D a *\left\{2\left[\left(\frac{\partial U}{\partial X}\right)^{2}+\left(\frac{\partial V}{\partial Y}\right)^{2}\right]+\left(\frac{\partial U}{\partial Y}+\frac{\partial V}{\partial X}\right)^{2}\right\}\right]$

$S_{\text {Total }}=S_{\theta}+S_{\psi}$

Note that, the viscous dissipation model as proposed by $\mathrm{Al}$ Hadhrami et al.(2003) is employed in Eq. (14). It may be noted that, the effect of viscous dissipation is neglected in the energy equation (Eq. (11)), but that is considered for estimation of $S_{\psi}$. Here $S_{\theta}$ and $S_{\psi}$ are local entropy generations due to heat transfer and fluid friction, respectively. In the above equation, $\xi$ is called the irreversibility distribution ratio, which is defined as $\xi=\frac{\mu T_{0}}{k}\left(\frac{\alpha}{K \Delta T^{2}}\right)^{2}=10^{-2}$ ( adopted from Varol et.al( 2009) and this is based on the order of magnitude estimation for all the numerical simulations. $\mathrm{T}_{0}$ is the bulk mean temperature and is considered to be $\left(\mathrm{T}_{\mathrm{h}}+\mathrm{T}_{\mathrm{c}}\right) / 2$.

\subsection{Local and Average Nusselt Number}

The heat transfer coefficient in terms of the local Nusselt number $(\mathrm{Nu})$ is defined by

$N u=-\frac{\partial \theta}{\partial n}$ where $\mathrm{n}$ denotes the normal direction on a plane.

$N u_{b}=-\frac{\partial \theta}{\partial Y}$

The average Nusselt number at the bottom and inclined side walls are given by:

$\overline{N u_{b}}=\frac{\int_{0}^{1} N u_{b} d X}{X_{0}^{1}}=\int_{0}^{1} N u_{b} d X$

\subsection{Bejan Number}

An alternative irreversibility distribution parameter is Bejan number $(\mathrm{Be})$, which is the ratio of heat transfer irreversibility $\left(\mathrm{HT}_{\mathrm{i}}\right)$ to global entropy generation rate (please refer Bejan (1996)).

$$
B e_{\text {avg }}=\frac{H T_{i}}{H T_{i}+F F_{i}}=\frac{S_{\theta \max }}{S_{\theta \max }+S_{\psi \max }}
$$

$\mathrm{Be}>>0.5$ is the limit at which, heat transfer irreversibility dominates; $\mathrm{Be}<<0.5$ is the opposite limit at which, irreversibility is dominated by fluid friction effects; and $\mathrm{Be} \sim 0.5$ is the case wherein, $\mathrm{HT}_{\mathrm{i}}$ and $\mathrm{FF}_{\mathrm{i}}$ are of equal significance.
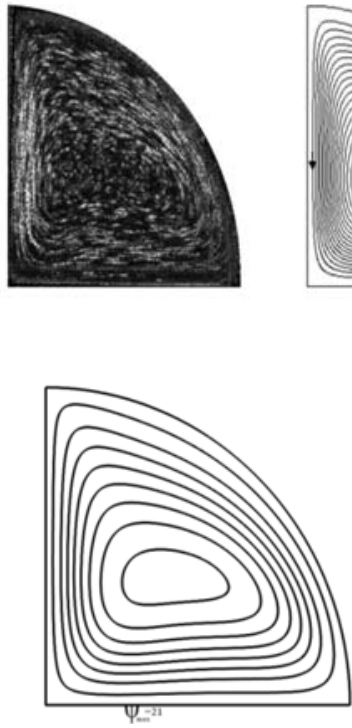

(a)

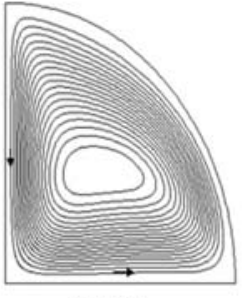

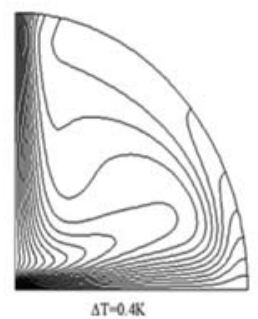

$\Delta T-0.4 \mathrm{~K}$

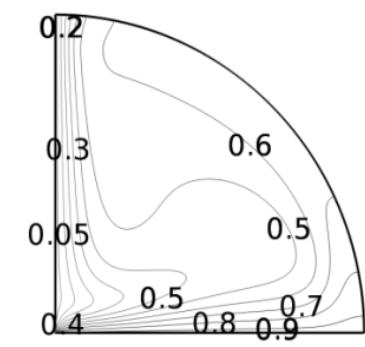

(b)

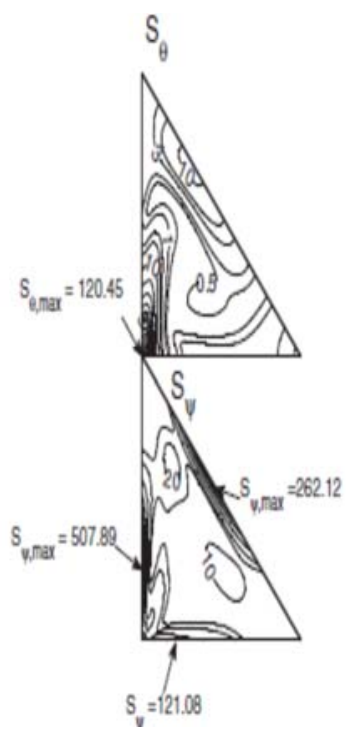

(c)

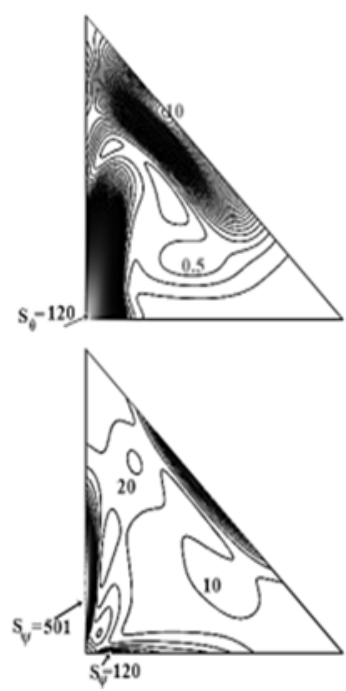

(d)
Fig. 2 (a) Experimental (left) and numerical (right) streamline and isotherm for $\mathrm{Ra}=1.7 \times 10^{5}$ (top row) adapted from (2011a); (b)Present Numerical Scheme with $\mathrm{Pr}=6.62$ : isotherms and streamline for $\mathrm{Ra}=$ $1.7 \times 10^{5}$ (bottom row), (c) Local entropy generation due to heat transfer $\left(S_{\theta}\right)$ and fluid friction $\left(S_{\psi}\right)$ contour plots for (linearly heated left wall), at $\operatorname{Pr}=7, R a=10^{5}$ for $\phi=45^{\circ}$ adapted from(2012); and (d) Present numerical scheme with $\operatorname{Pr}=7, R a=10^{5}$ :Local entropy generation due to heat transfer $\left(S_{\theta}\right)$ and fluid friction $\left(S_{\psi}\right)$ contour plots. 


\section{CODE VALIDATION AND GRID INDEPENDENCY TEST}

Due to the lack of suitable results in the literature pertaining to the present porous configuration, the result obtained have been validated against the existing results for a quadrantal cavity filled with a water medium $(\mathrm{Pr}=6.62)$ when the bottom wall is heated and the vertical wall is cold. ( refer to Aydin and Yesiloz, (2011). Figure 2a,b clearly indicate that the results are in good agreement with the prior published data. Further, entropy generation results were not available for this type of enclosure so the validation was carried out for results obtained from triangular enclosure(Basak et.al(2012).Figure 2c,d also indicate that the results are in good agreement with the prior published data.

Grid Independency test was also carried out for average Nusselt number for bottom wall for $\operatorname{Pr}=0.71$ at $\mathrm{Da}=10^{-3}$ for this present configuration and final results are depicted for Ra-46018 elements. In the study, three different mesh sizes (21612 finite elements, 46018 finite elements, and 63366 finite elements) are adopted in order to check the mesh independence. A detailed grid independence study has been performed, and the results are obtained for the average Nusselt number for bottom wall and they are represented in Table 1, but no considerable changes were observed for average Nusselt number values for the bottom wall. Thus, a grid size of 46018 finite elements is found to meet the requirements of both the grid independency study and also because on further refinement of grids the change in the average Nusselt number is almost negligible( less than $1 \%$ ).

Table 1: Average Nusselt Number for Bottom wall values vs $\mathrm{Ra}\left(\mathrm{Da}=10^{-}\right.$ 3)

\begin{tabular}{|c|c|c|c|}
\hline $\mathrm{Ra}$ & $\begin{array}{c}\mathrm{Nu}_{\text {Avg }} 63366 \\
\text { Elements }\end{array}$ & $\begin{array}{c}\mathrm{Nu}_{\text {Avg }} 46018 \\
\text { Elements }\end{array}$ & $\begin{array}{c}\mathrm{Nu}_{\text {Avg }} 21612 \\
\text { Elements }\end{array}$ \\
\hline $10^{6}$ & 6.23 & 6.22 & 6.20 \\
\hline $10^{5}$ & 2.56 & 2.55 & 2.54 \\
\hline $10^{4}$ & 1.89 & 1.88 & 1.88 \\
\hline
\end{tabular}

\section{NUMERICAL SOLUTION METHODOLOGY}

The governing transport equations (Eqs.5-8) together with the boundary conditions (Eqs 1-4) as outlined in the previous section are solved numerically using finite element method. The Galerkin weighted method is used to transform the governing equations into a system of integral equations. The detailed description of the methodology can be found in Zienkiewicz and Taylor (1991). The Gauss's quadrature method (refer Zienkiewicz and Taylor (1991)) is also used to perform the integration. The Newton-Raphson technique is employed to solve the set of algebraic equations. The convergence criterion is set such as $\max \left|\phi^{n+1}-\phi^{n}\right| / \phi^{n} \leq 10^{-6}$ where $\phi$ represents any transport variable.

\section{RESULTS AND DISCUSSION}

Numerical simulations are performed to ascertain fluid flow, heat transfer and entropy generation characteristics in this enclosure for this particular study. We first analyse the stream lines and Isotherms and later discuss about entropy generation in the quadrantal enclosure.

\subsection{Stream lines and Isotherms}

Heat transfer and flow distributions are presented via streamlines in Figure 3-5 and isotherms in Figures. 6-8. The streamlines and isotherm results are depicted for $\mathrm{Ra}=1 \times 10^{4}, 1.7 \times 10^{5}$ and $1 \times 10^{6}$ for $\mathrm{Da}=10^{-5}, 10^{-4}$ and $10^{-3}$.

Examining Figures 3(a-c), 4(a,c) \& 5(a-c) it is also observed that the difference in temperature between the inner and outer walls creates density variations within the fluid filling the porous enclosure and gives rise to the buoyancy forces that move the flow. The thermo physical parameters in the problem are Rayleigh number (Ra),the Prandtl number (Pr), and the Darcy number (Da). From Figure 3(a) for the following Darcy number of $\mathrm{Da}=10^{-5}$, the strength of the vortex is negligibly small when $\mathrm{Ra}=10^{4}$ because of combined confluence of high resistance to flow and low buoyancy force and porous drag. With the increase in Rayleigh number, the buoyancy force increases as a result of which the strength of the vortices rotating in both clockwise and anticlockwise direction are enhanced. For example, the maximum strength of vortex at $\mathrm{Ra}=10^{4}$ is 0.021 in both clockwise and anticlockwise direction and the value increases to 0.35 and -0.35 respectively with increase of $R$ a to $1.7 \times 10^{5}$ in Figure 3(b) .With a further increase of $\mathrm{Ra}=10^{6}$ in Figure 3(c) we find that the stream line circulation strength has increased to 2.1. Thus we can say that the effect of circulation strength is low at low permeability of the working fluid.

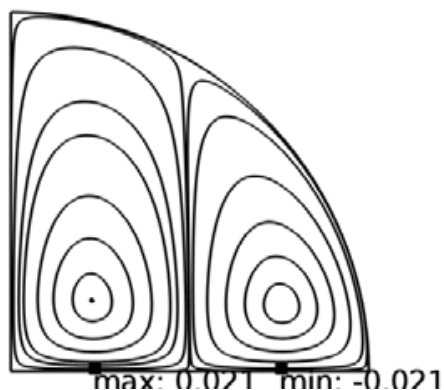

(a)



(b)

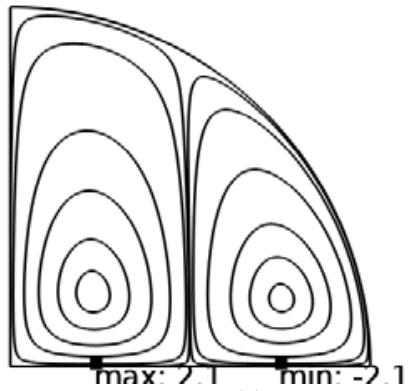

(c)

Fig. 3 Streamlines from the present study(a) $\mathrm{Ra}=1 \times 10^{4}$ (b) $\mathrm{Ra}=$ $1.7 \times 10^{5}$, (c) $\mathrm{Ra}=1 \times 10^{6} ; \mathrm{Da}=10^{-5}, \operatorname{Pr}=0.71$

In Figure 4 at higher $\mathrm{Da}$ number of $\mathrm{Da}=10^{-4}$ at low $\mathrm{Ra}$ we observe similar trends of circulation pattern and strength at low $\mathrm{Ra}=10^{4}$. When $\mathrm{Ra}$ is increased from $1.7 \times 10^{5}$ and $10^{6}$, we observe that the stream line pattern doesn't alter in visualisation but there is a sufficient increase of stream line strength at $\mathrm{Ra}=10^{6}$ and $\psi_{\max }=22$ for the anticlockwise roll. So what we observe for permeability of fluid for $\mathrm{Da}=10^{-4}$ for Figure 4(a-c) 
that the quality and magnitude of stream lines are quite similar to that for permeability of fluid for $\mathrm{Da}=10^{-5}$ and only when we increase the $\mathrm{Ra}=10^{6}$ there is a major increase of circulation strength of flow in both anticlockwise and clockwise direction proving that convection phenomenon is predominant at this particular Rayleigh number. With a further increase of Darcy number from $10^{-4}$ to $10^{-3}$ in Figure $5(\mathrm{a}-\mathrm{c})$ the strength of stream function increases and more over the streamlines that comprise the major circulation cell continue to occupy more than $75 \%$ of the enclosure. Further the streamline patterns that are observed in the enclosure are found to be elliptical in shape which results with distinct boundary layers, and thus as a consequence, the energy transport is enhanced .It is also observed at Figure 5(c) for $\mathrm{Da}=10^{-3}, \mathrm{Ra}=10^{6}$, that the phenomenon of the dominant buoyancy forces leads to enhanced circulation, as depicted from the larger magnitudes of stream functions $|\psi| \max _{\max }>112$ for (for the anticlockwise direction flow). Note that increase in the magnitude of the stream functions $(\psi)$ signify that the buoyancy forces start to dominate over viscous forces. Thus with an increase in the Darcy number this indicates either an increase in the permeability of the porous matrix or corresponding increase in transport of fluid.

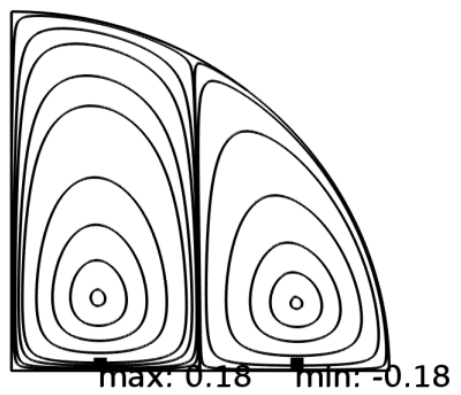

(a)

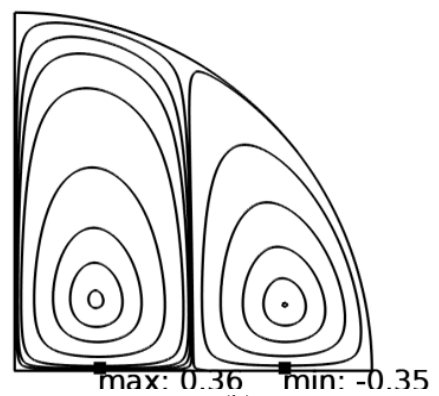

(b)

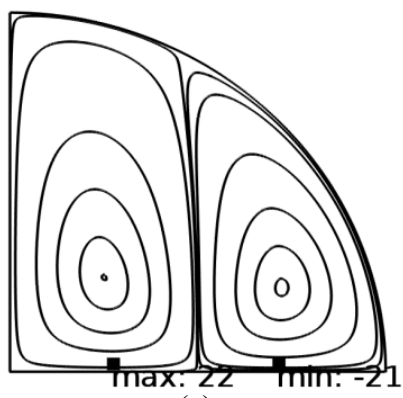

(c)

Fig. 4 Streamlines from the present study(a) $\mathrm{Ra}=1 \times 10^{4}$ (b) $\mathrm{Ra}=$ $1.7 \times 10^{5}$, (c) $\mathrm{Ra}=1 \times 10^{6} ; \mathrm{Da}=10^{-4}, \mathrm{Pr}=0.71$

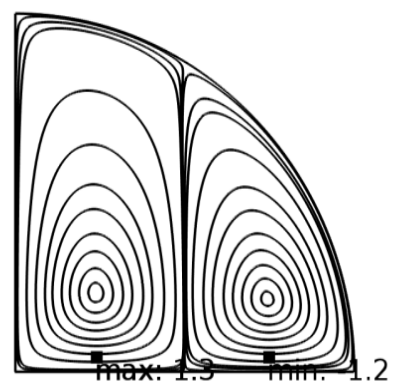

(a)

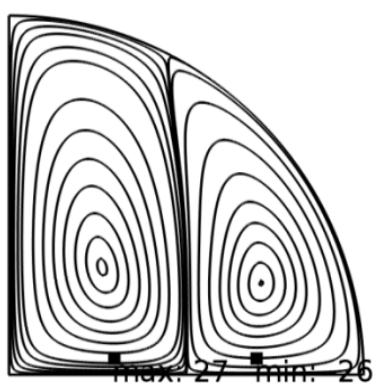

(b)



(c)

Fig. 5 Streamlines from the present study(a) $\mathrm{Ra}=1 \times 10^{4}$ (b) $\mathrm{Ra}=$ $1.7 \times 10^{5}$, (c) $\mathrm{Ra}=1 \times 10^{6} ; \mathrm{Da}=10^{-3}, \mathrm{Pr}=0.71$

Isotherms for the following study are depicted for Figure 6-8,(a-c). It is also found that higher value isotherms are located on the mid region of the enclosure because of the imposed temperature gradient on the bottom wall. Also the isotherm patterns are asymmetrical because of the non-uniform heating of the enclosure at higher Darcy number compared to lower Darcy number. At lower Rayleigh values and lower Darcy values in Figure 6(a-b) \& 7(a), the nature of isotherm clearly indicates that heat is transferred mainly due to conduction because of the fact that resistance due to porous drag dominates over the buoyancy force. An increase in the permeability of the porous matrix implies lower hydrodynamic resistance and consequently stronger convective flow and this phenomenon proves the fact for the nature of isotherm patterns for Figure7(c) \& 8 (b-c) at higher Rayleigh numbers .Further the effect of asymmetry is more profound for $\mathrm{Da}=10^{-3}$ rather than $\mathrm{Da}=10^{-5}$ because of enhanced convection. 


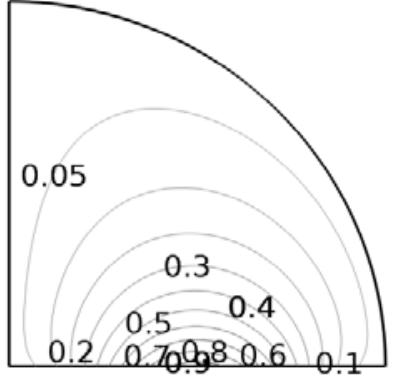

(a)

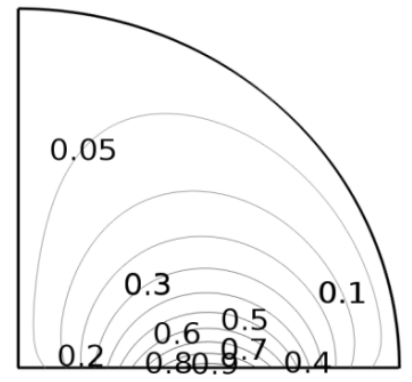

(b)

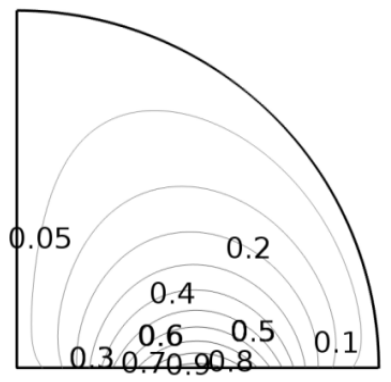

(c)

Fig. 6 Isotherms from the present study(a) $\mathrm{Ra}=1 \times 10^{4}$ (b) $\mathrm{Ra}=1.7 \times 10^{5}$, (c) $\mathrm{Ra}=1 \times 10^{6} ; \mathrm{Da}=10^{-5}, \operatorname{Pr}=0.71$

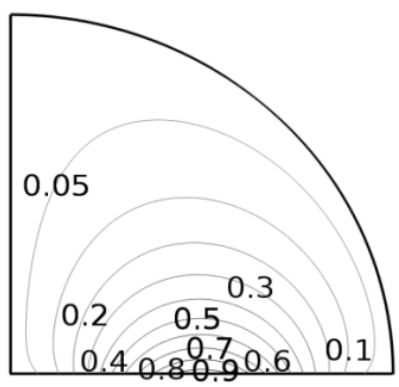

(a)



(b)

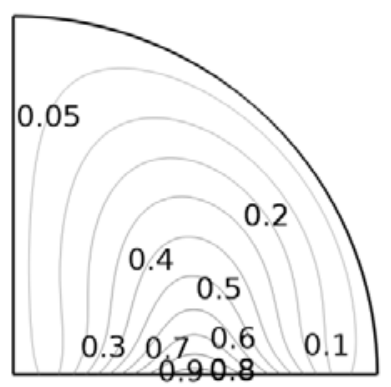

(c)

Fig. 7 Isotherms from the present study(a) $\mathrm{Ra}=1 \times 10^{4}$ (b) $\mathrm{Ra}=1.7 \times 10^{5}$, (c) $\mathrm{Ra}=1 \times 10^{6} ; \mathrm{Da}=10^{-4}, \mathrm{Pr}=0.71$

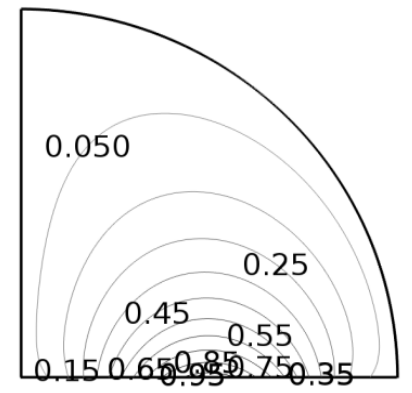

(a)



(b)

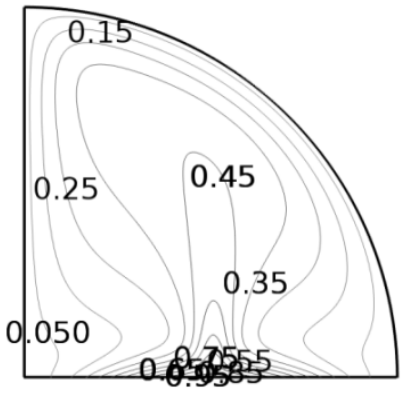

(c)

Fig. 8 Isotherms from the present study(a) $\mathrm{Ra}=1 \times 10^{4}$ (b) $\mathrm{Ra}=1.7 \times 10^{5}$, (c) $\mathrm{Ra}=1 \times 10^{6} ; \mathrm{Da}=10^{-3}, \mathrm{Pr}=0.71$

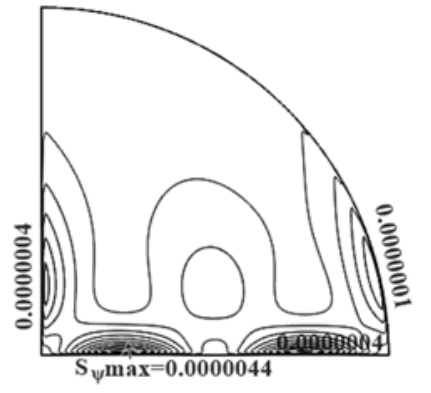

(a)

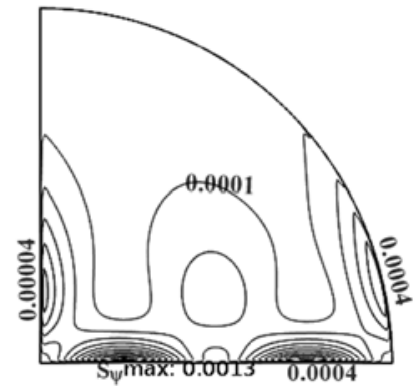

(b)

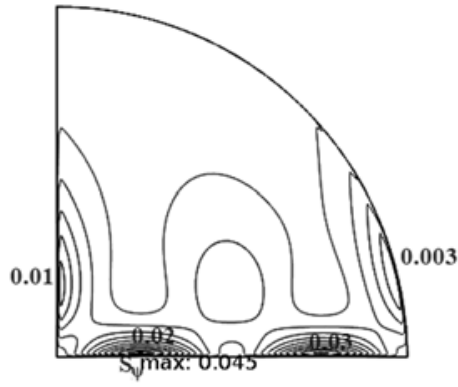

(c)

Fig. 9 Local entropy generation due to fluid friction from the present study(a) $\mathrm{Ra}=1 \times 10^{4}$ (b) $\mathrm{Ra}=1.7 \times 10^{5},(\mathrm{c}) \mathrm{Ra}=1 \times 10^{6} ; \mathrm{Da}=10^{-5}, \operatorname{Pr}=0.71$ 


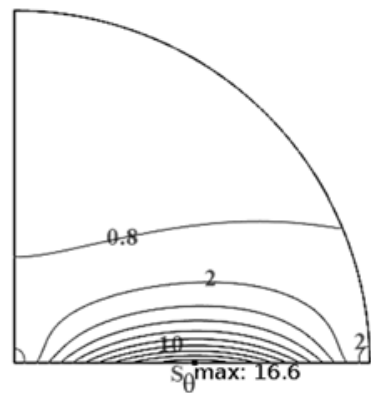

(a)

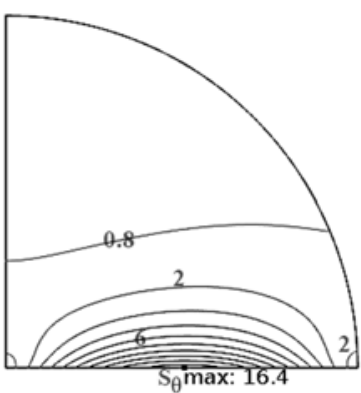

(b)

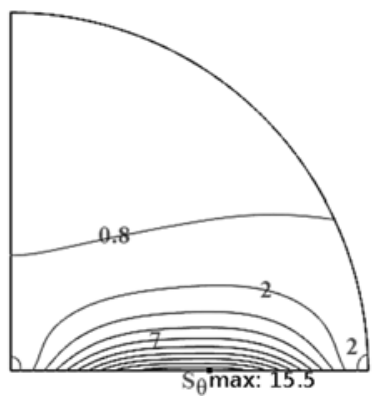

(c)

Fig. 10 Local entropy generation due to heat transfer from the present study(a) $\mathrm{Ra}=1 \times 10^{4}$ (b) $\mathrm{Ra}=1.7 \times 10^{5},(\mathrm{c}) \mathrm{Ra}=1 \times 10^{6} ; \mathrm{Da}=10^{-5}, \mathrm{Pr}=0.71$

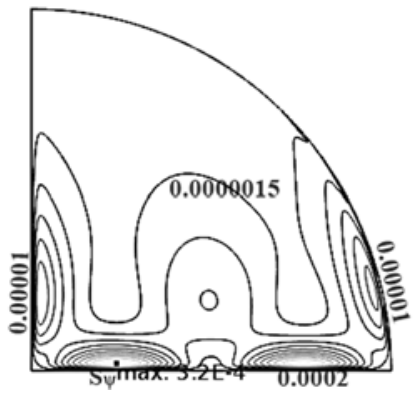

(a)

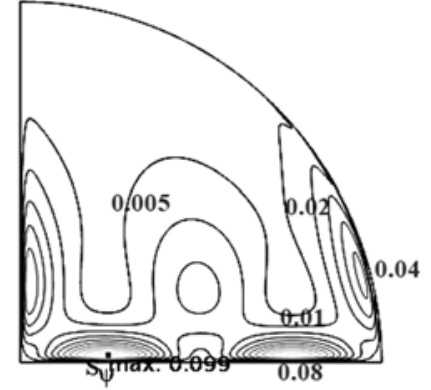

(b)

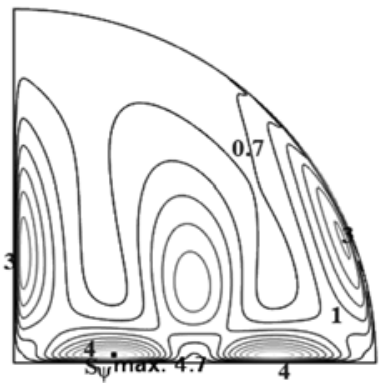

(c)

Fig. 11 Local entropy generation due to fluid friction from the present study(a) $\mathrm{Ra}=1 \times 10^{4}$ (b) $\mathrm{Ra}=1.7 \times 10^{5},(\mathrm{c}) \mathrm{Ra}=1 \times 10^{6} ; \mathrm{Da}=10^{-4}, \operatorname{Pr}=0.71$

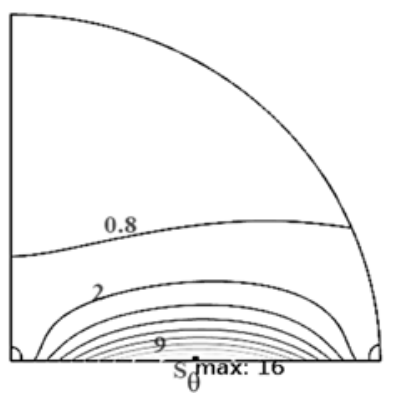

(a)

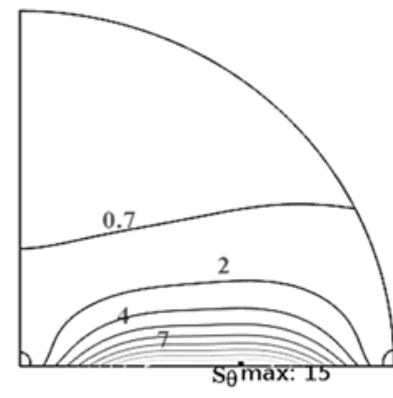

(b)



(c)

Fig. 12 Local entropy generation due to heat transfer from the present study(a) $\operatorname{Ra}=1 \times 10^{4}$ (b) $\operatorname{Ra}=1.7 \times 10^{5},(\mathrm{c}) \mathrm{Ra}=1 \times 10^{6} ; \mathrm{Da}=10^{-4}, \operatorname{Pr}=0.71$

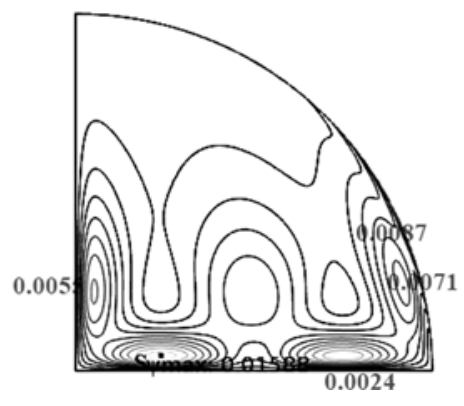

(a)



(b)

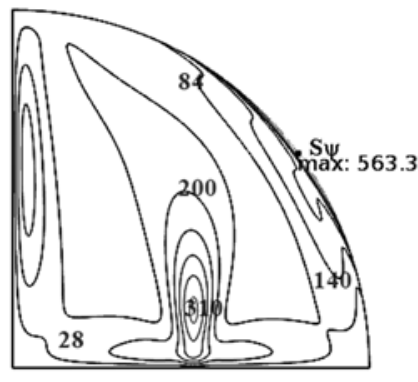

(c)

Fig. 13 Local entropy generation due to fluid friction from the present study(a) $\mathrm{Ra}=1 \times 10^{4}$ (b) $\mathrm{Ra}=1.7 \times 10^{5},(\mathrm{c}) \mathrm{Ra}=1 \times 10^{6} ; \mathrm{Da}=10^{-3}, \mathrm{Pr}=0.71$ 


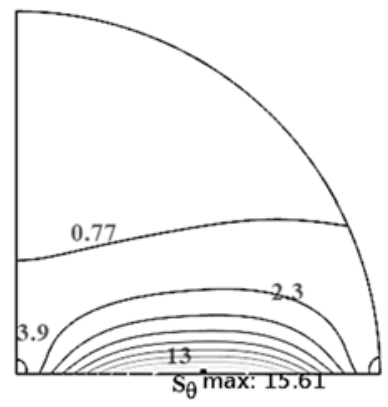

(a)



(b)

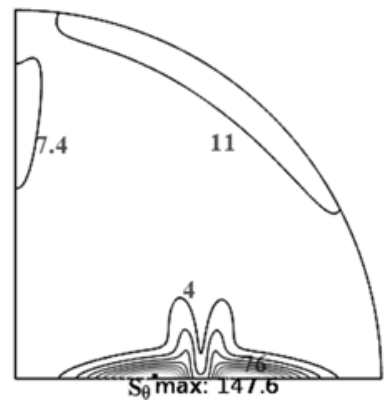

(c)

Fig 14 Local entropy generation due to heat transfer from the present study(a) $\operatorname{Ra}=1 \times 10^{4}$ (b) $\mathrm{Ra}=1.7 \times 10^{5},(\mathrm{c}) \mathrm{Ra}=1 \times 10^{6} ; \mathrm{Da}=10^{-3}, \mathrm{Pr}=0.71$

\subsection{Entropy Generations}

Figures.9-14 (a), (b) and (c) depicts the entropy generation due to fluid friction and heat transfer irreversibility inside the enclosure for $\mathrm{Da}=10^{-5}$ to $\mathrm{Da}=10^{-3}$. The entropy generation values obtained for fluid friction for this case, i.e for $\mathrm{Da}=10^{-5}$ is practically negligible inside the enclosure. The maximum values are found to be ranging between $S_{\psi \max }=4 \times 10^{-6}$ (for $\mathrm{Ra}=10^{4}$ ) to $\mathrm{S}_{\psi \max }=.045$ (for $\mathrm{Ra}=10^{6}$ ) for Figure $9(\mathrm{a}-\mathrm{c})$. It is also observed that entropy generation due to heat transfer is also negligible in the core region and also it has very less values for $\mathrm{Ra}=10^{4}, \mathrm{Da}=10^{-5}$ as observed from Figure 10(a). As we increase the Rayleigh number from $\mathrm{Ra}=10^{4}$ to $1.7 \times 10^{5}$ in Fig 10(b) and then further to $10^{6}$ in Fig 10(c),entropy generation due to heat transfer decreases slightly or practically remains the same (i.e from $\mathrm{S}_{\max }=16.6$ to $\mathrm{S}_{\theta \max }=15.5$ ) for $\mathrm{Da}=1 \times 10^{-5}$ because of the conduction like situation though there has been an significant increase of Rayleigh number.

Figure $11 \& 12$ (a), (b) and (c) depicts the entropy generation due to fluid friction and heat transfer irreversibility inside the enclosure for $\mathrm{Da}=10^{-4}$. The corresponding entropy generation for fluid friction for this case, i.e $\mathrm{Da}=10^{-4}$ for Figure 11(a) is also practically negligible inside the enclosure with maximum values ranging between $S_{\psi \max }=3.2 \times 10^{-4}$ (for $\mathrm{Ra}=10^{4}$ ) to $\mathrm{S}_{\psi \max } 4.7\left(\right.$ for $\mathrm{Ra}=10^{6}$ ) found in Figure 11(c). It is also observed that, here also the dominant source of irreversibility is due to heat transfer. The values observed are $\mathrm{S}_{\theta \max }=23$ (bottom wall) (for $\mathrm{Ra}$ $=10^{6}, \mathrm{Da}=10^{-4}$ ) and is found in Figure 11(c). So it is observed that the entropy generation values of heat transfer irreversibility are practically same or having very less change when we increase the Da number from $10^{-5}$ to $10^{-4}$. On the other hand what we also observe is negligible entropy generation production due to fluid flow irreversibility.

Figures 13 \& 14 (a), (b) and (c) depicts the entropy generation due to fluid friction and heat transfer irreversibility inside the enclosure for $\mathrm{Da}=10^{-3}$.It is observed that the dominant source of irreversibility for this case is due to fluid friction. For lower $\mathrm{Ra}=10^{4} \mathrm{~S}_{\psi}<\mathrm{S}_{\theta}$ and this is found in Figure 13( a) \& 14 (a) respectively. However with increase of $\mathrm{Ra}=1.7 \times 10^{5} \mathrm{~S}_{\psi \max }=12.12$ ( location at right curved cold wall) found in Fig 13(b) the values are found to have increased and also the values reported are approaching to nearly half of the values of entropy generation irreversibility due to heat transfer. $S_{\theta \max }=30$ (bottom wall) (for $\left.\mathrm{Ra}=1.7 \times 10^{5}, \mathrm{Da}=10^{-3}\right)$ and found in Figure 14(b). There is a significant increase in the entropy generation due to fluid friction when Rayleigh number changes it value from $1.7 \times 10^{5}$ to $10^{6}$ and found in Figure 13(c).This is due to a smaller thermal gradient near the side cold wall and also because of higher thermal mixing near the core of the cavity in nonlinear heating. Convection effects are prominent in this case specially at higher Rayleigh numbers and for this case $S_{\psi \max }($ value=563) and this is much higher than corresponding $S_{\theta \max }\left(v^{2} a l u e=148\right)$ found in Figure14(c) for $\mathrm{Ra}=10^{6}$.

\subsection{Nusselt Number}



(a)

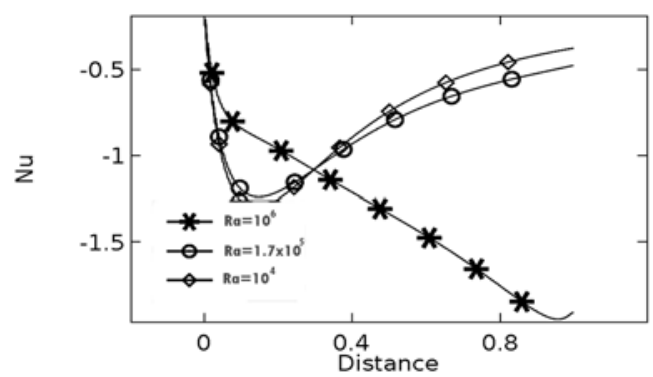

(b)

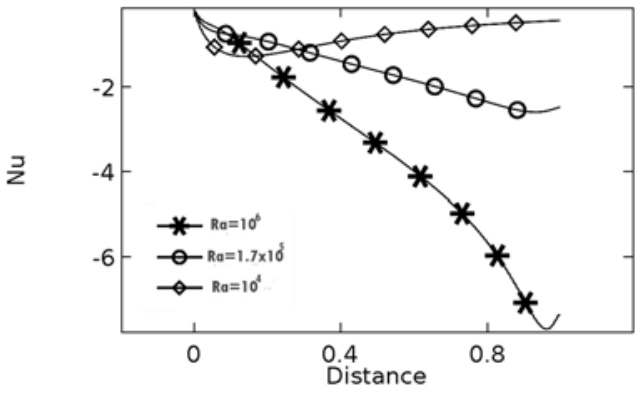

(c)

Fig. 15 Local Nusselt number on the cold vertical wall versus Rayleigh number(a) $\mathrm{Da}=10^{-5}$ (b) $\mathrm{Da}=10^{-4}$ (c) $\mathrm{Da}=10^{-3}$ 




(a)

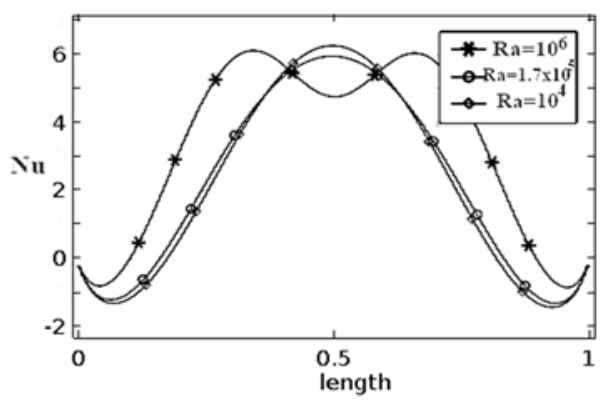

(b)

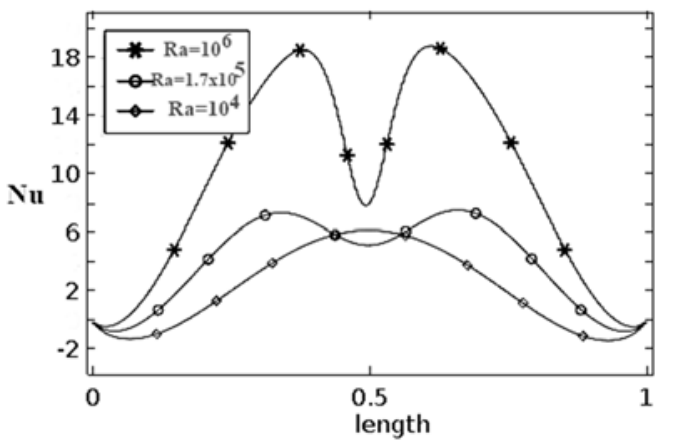

(c)

Fig. 16 Local Nusselt number on the hot bottom wall versus Rayleigh number(a) $\mathrm{Da}=10^{-5}$ (b) $\mathrm{Da}=10^{-4}$ (c) $\mathrm{Da}=10^{-3}$

The effect of Ra on the local Nusselt number for cold wall is demonstrated in Fig. 15, while the local Nusselt number variation with $\mathrm{Ra}$ for hot bottom wall is shown in Figure 16. Overall at low Rayleigh numbers $\mathrm{Da}=10^{-5} \mathrm{Ra}=10^{4}$ the influence of Rayleigh number on the local Nusselt number is not significant. The influence becomes stronger as the Rayleigh number increases beyond $10^{4}$. The poor heat transfer performance at low $\mathrm{Ra}$ numbers can be attributed to the weaker convection which is very clear from the panel plots of local Nusselt number for different Da number and all values reported are negative as observed for Figures 15(a-c). Maximum influence of $\mathrm{Ra}=10^{6}$ is only prominent in the panel plot for $\mathrm{Da}=10^{-3}$. The value is approaching towards the positive side at the right hand part of enclosure (see panel plots corresponding to figure 15(c) .

For analysis of local Nusselt number for the hot bottom wall we refer to Figure $16(\mathrm{a}-\mathrm{c})$. What we observe is that, the value is $\sim 18$ at two points for the panel plots corresponding to Figure 16(c) for $\mathrm{Ra}=10^{6}$ and corresponding $\mathrm{Da}=10^{-3}$. There is a local dip in values for local Nusselt number for bottom wall and they show very less values near the end of enclosure. The Nusselt number slightly increases again around the corner point and also continues to increase along the hot wall in order that temperature difference between the hot wall and the adjacent fluid layer decreases along the y-direction and also justifies the nature of temperature profile imposed on the wall.



(a)

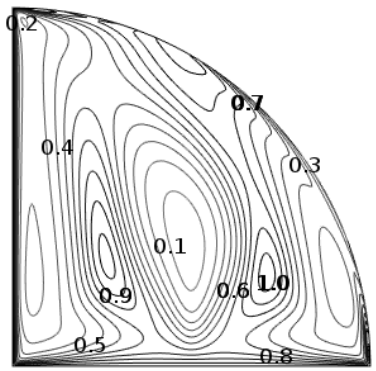

(b)

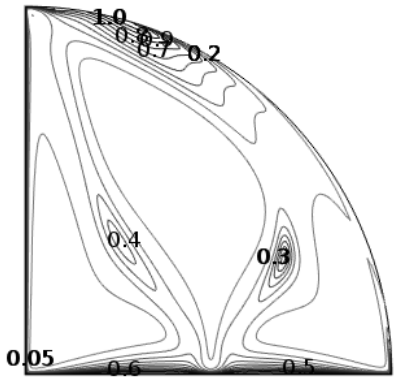

(c)

Fig 17 Bejan contour plots due to total entropy generation from the present study(a) $\mathrm{Ra}=1 \times 10^{4}$ (b) $\mathrm{Ra}=1.7 \times 10^{5}$, (b) $\mathrm{Ra}=1 \times 10^{6}$; $\mathrm{Da}=10^{-3}, \operatorname{Pr}=0.71$

Table 2: Total entropy generation rate and average Bejan number at different inclination angle for $\mathrm{Ra}=10^{6}, 10^{4}, \mathrm{Da}=10^{-3}$

\begin{tabular}{|l|l|l|l|l|}
\hline $\mathrm{Da}=10^{-3}$ & $\mathrm{~S}_{\theta, \max }$ & $\mathrm{S}_{\psi, \max }$ & $\mathrm{S}_{\mathrm{T}}$ & Beavg \\
\hline $\mathrm{Ra}=10^{6}$ & 147.97 & 563.3 & 711.27 & 0.208 \\
\hline $\mathrm{Ra}=10^{4}$ & 16 & 0.016 & 16.016 & 0.999 \\
\hline
\end{tabular}

From the Bejan plots in Figure 17 (a-c) and Bejan number values that are found in Table 2 respectively for it's quite clear that the heat transfer irreversibility dominates at lower Ra when convection is poor and fluid friction irreversibility is more at higher Ra when convection effects are predominant .

\section{CONCLUSIONS}

Natural convection in a quadrantal enclosure filled with porous material has been studied numerically. The main conclusions are:

1) As the Darcy number increases from $\mathrm{Da}=10^{-5}$ to $\mathrm{Da}=10^{-3}$ and Rayleigh number $(\mathrm{Ra})$ increases to $10^{6}$, the fluid flow as well as thermal energy transport intensifies, because of enhanced convection. Consequently, the entropy generation due to heat $\operatorname{transfer}\left(\mathrm{s}_{\theta}\right)$ and fluid friction $\left(\mathrm{S}_{\psi}\right)$ also increases. 
2) The entropy generation due to heat transfer $\left(\mathrm{S}_{\theta}\right)$ is significant in the bottom half of the enclosure near bottom wall, because of a large temperature gradient, whereas $S_{\psi}$ is significant in the portions where velocity gradient is large, especially where the solid wall is in contact with the adjacent circulation cells.

3) The significant contributor of entropy generation is nearly similar for both Darcy numbers i.e. $\mathrm{Da}=10^{-5}$ and $10^{-4}$ and occurs because of heat transfer irreversibility whereas the fluid friction irreversibility is practically negligible for both these two cases of Darcy number.

4) Fluid friction irreversibility dominates over heat transfer irreversibility for $\mathrm{Da}=10^{-3} \mathrm{Ra}=10^{6}$.

5) Local Nusselt Number values are more, numerically only for $\mathrm{Ra}=10^{6}, \mathrm{Da}=10^{-3}$ for cold wall as well as hot wall.

6) Based on EGM analysis and Bejan number analysis, it is established that total entropy production is not significant with larger thermal mixing at high Darcy number $\left(\mathrm{Da}=10^{-3}\right)$ and maybe recommended as for this type of enclosure discussed in the present study.

\section{NOMENCLATURE}

$\mathrm{c}_{\mathrm{p}} \quad$ specific heat

Da Darcy number

g acceleration due to gravity

$\mathrm{H}$ enclosure height

$\mathrm{K}$ thermal conductivity

K Permeability

$\mathrm{L} \quad$ enclosure length

$\mathrm{N}_{\mathrm{u}} \quad$ Nusselt number

$\mathrm{P} \quad$ pressure

$\mathrm{P} \quad$ non-dimensional pressure

Pr Prandtl number

$\mathrm{Ra} \quad$ Rayleigh number

$\mathrm{S} \quad$ dimensionless total entropy generation

$\mathrm{S}_{\theta} \quad$ dimensionless entropy generation due to heat transfer

$\mathrm{S}_{\psi} \quad$ dimensionless entropy generation due to fluid friction

S total dimensionless total entropy generation due to heat transfer and fluid friction

$\mathrm{T}$ temperature

$\mathrm{u} \quad$ velocity component in the $\mathrm{x}$-direction

$\mathrm{v} \quad$ velocity component in the v-direction

$\mathrm{U}$ non-dimensional velocity component in the $\mathrm{x}$-direction

$\mathrm{V}$ non-dimensional velocity component in the y-direction

$\mathrm{x}, \mathrm{y} \quad$ Cartesian coordinate system

$\mathrm{X}, \mathrm{Y}$ non-dimensional coordinates

\section{Greek Symbols}

$\Psi \quad$ non-dimensional stream function $(=\psi / \alpha)$

$\psi \quad$ stream function

$\rho$ density

$\beta \quad$ co-efficient of thermal expansion

$v \quad$ kinematic viscosity

$\alpha \quad$ thermal diffusivity

$\mu \quad$ dynamic viscosity

\section{Subscripts}

c cold wall

h hot wall

max maximum

min minimum

\section{REFERENCES}

Al-Hadhrami, A.K., Elliott, L., and Ingham, D.B.,(2003), “A New Model for Viscous Dissipation in Porous Media across a Range of Permeability Values", Transport in Porous Media , 53, 117- 122.

Aydin, O. and Yesiloz, G.,(2011), "Natural Convection in a Quadrantal Cavity Heated and Cooled on Adjacent Walls", Journal of Heat Transfer, 133, 052501-052507.

http://dx.doi.org/10.1115/1.4003044

Bejan,A.,(1979), "On the Boundary Layer Regime in a Vertical Enclosure filled with a Porous Medium", Letters. Heat Mass Transfer,6, 93-102.

Bejan,A.,(1996),“Entropy Generation Minimization”, CRC, Boca Raton New York.

Bondareva, N.S.,Sheremet, M.A., Oztop, H.F., and Abu-Hamdeh, N., (2017), "Entropy Generation Due to Natural Convection of a Nanofluid in a Partially Open Triangular Cavity", Advanced Powder Technology,28 244-255.

http://dx.doi.org/10.1016/j.apt.2016.09.030

Basak ,T., Anandalakshmi, R., and Gunda, P.,(2012),"Role of Entropy Generation during Convective Thermal Processing in Right-Angled Triangular Enclosures with Various Wall Heatings", Chemical Engineering Research and Design 90, 1779-1799.

http://dx.doi.org /10.1016/j.cherd.2012.03.008

Basak,T., Singh, A. K., Richard, R. and Roy, S.(2013), "Finite Element Simulation with Heatlines and Entropy Generation Minimization During Natural Convection within Porous Tilted Square Cavities", Ind. Eng. Chem. Res.,52, 8046-8061.

http://dx.doi.org / 10.1021/ie4005755

Basak,T., Kaluri,R.S. and Balakrishnan, A.R.,(2012), "Entropy Generation During Natural Convection in a Porous Cavity: Effect of Thermal Boundary Conditions, Numerical. Heat Transfer A, 62, 336364.

http://dx.doi.org/10.1080/10407782.2012.691059

Bhuvaneswari, M., Sivasankaran,S., and Kim, Y. J., (2011),"Magneto Convection in a Square Enclosure with Sinusoidal Temperature Distributions on Both Side Walls", Numerical Heat Transfer- A,59,167184.

http://dx.doi.org/10.1080/10407782.2011.541219.

Bose, P. K.,Sen.D., Panua, R. and Das,A.(2013),"Numerical Analysis of Laminar Natural Convection in a Quadrantal Cavity with a Solid Adiabatic Fin Attached to the Hot Vertical Wall", Journal of Applied. Fluid Mechanics.,6(4), 501-510.

Baytas, A.C. and Pop I.,(1999), "Free Convection in Oblique Enclosures filled with a Porous Medium", International Journal of Heat and Mass Transfer",42(6),1047-1057.

http://dx.doi.org/10.1016/S0017-9310(98)00208-7

Baytas , A.C., (2000), "Entropy Generation for Natural Convection in an Inclined Porous Cavity", International Journal of Heat and Mass Transfer, 43, 2089-2099.

Chou, H.M.,Wu, H.W, Lin, I.H, Yang, W.J, and Cheng, M.L. (2015), "Effects of Temperature-Dependent Viscosity on Natural Convection in Porous Media", Numerical. Heat Transfer -A, 68, 1331-1350. 


\section{http://dx.doi.org/10.1080/10407782.2015.1012864}

Dalal A. and Das, M. K., (2005), "Laminar Natural Convection in an Inclined Complicated Cavity with Spatially Variable Wall Temperature", International Journal of Heat and Mass Transfer, 48, 3833-3854.

http://dx.doi.org/10.1016/j.ijheatmasstransfer.2004.07.051.

Dutta, S., Biswas, A. and Pati, S.,(2018), "Natural convection heat transfer and entropy generation inside porous quadrantal enclosure with non-isothermal heating at the bottom wall', Numerical Heat Transfer, Part A: Applications, 73, 1-19.

http://doi.org/10.1080/10407782.2018.1423773.

Gibanov, N.S., Sheremet, M.A., Oztop, H. F., and Al-Salem K., (2017), "Effect of Uniform Inclined Magnetic Field on Natural Convection and Entropy Generation in an Open Cavity having a Horizontal Porous Layer saturated with a ferrofluid", Numerical Heat Transfer- A 72 (6), 479-494. http://dx.doi.org/ 10.1080/10407782.2017.1386515

Moya, S.L., Ramos, E., and Sen, M.,1987 "Numerical Study of Natural Convection in a Tilted Rectangular Porous Material", International Journal of Heat and Mass Transfer, 30, 741-756.

Moukalled F. and Darwish, M.,(2010),"Natural Convection Heat Transfer in a Porous Rhombic Annulus, Numerical Heat Transfer Part A, $\mathbf{5 8 , 1 0 1 - 1 2 4 . ~}$

http://dx.doi.org/ 10.1080/10407782.2010.497322.

Nield, D.A. and Bejan, A.,(2006),"Convection in Porous Media”, Springer-Verlag, New York.

Pandit, S.K., and Chattopadhyay,A.,(2014),"Higher Order Compact Computations of Transient Natural Convection in a Deep Cavity with Porous Medium", International Journal of. Heat and Mass Transfer, 75, 624-636.

http://dx.doi.org/10.1016/j.ijheatmasstransfer.2014.03.079

Prasad, V., and Kulacki. F.A.,(1984), "Convective Heat Transfer in a Rectangular Porous Cavity Effect of Aspect Ratio on Flow Structure and Heat transfer", Journal of Heat Transfer, 106, 158-165.

Sarris, I. E., Lekakis, I. and N. S. Vlachos, (2002), "Natural Convection in 2D Enclosure with Sinusoidal Upper Wall Temperature", Numerical Heat Transfer- A. 42, 513-530.

http://dx.doi.org/10.1080/10407780290059675.

Sen, D., Bose, P. K., Panua, R. and Das, A.(2013),"Laminar Natural Convection Study in a Quadrantal Cavity using Heater on Adjacent Walls, Frontiers in Heat and Mass Transfer, 4(1), 013005,1-7. http://dx.doi.org/ 10.5098/hmt.v4.1.3005
Sheremet, M., Pop.I., Öztop H. F.,and Abu-Hamdeh N.,.(2017), "Natural Convection of Nanofluid inside a Wavy Cavity with a Non-Uniform Heating: Entropy Generation Analysis", International. Journal of Numerical Methods and Heat Fluid Flow 27,958-980. http://dx.doi.org/10.1108/HFF-02-2016-0063

Sen, D., Bose, P. K., Panua, R. and Das, A.(2015),“ Numerical Analysis of Laminar Natural Convection in a Quadrantal Cavity with a Hot Bottom and Cold Curved Walls", Heat Transfer Research, 46(7), 631-641. http://dx.doi.org/ 10.1615/HeatTransRes.2015004629

Tiwari, A.K., Singh, A.K., Chandran,P. And Sacheti, N.C.,(2012), "Natural Convection in a Cavity with a Sloping Upper Surface filled with an Anisotropic Porous Material", Acta Mechanica,223, 95-108. http://dx.doi.org/ 10.1007/s00707-011-0544-5.

Vafai K. and Tien, C. L. ,(1981), "Boundary and Inertia Effects on Flow and Heat Transfer in Porous Media, International Journal of Heat and Mass Transfer, 24(2),195-203.

Varol, Y., Oztop, H. F. and Pop, I.,(2009), "Entropy Analysis due to Conjugate-Buoyant Flow in a Right-Angle Trapezoidal Enclosure Filled with a Porous Medium Bounded by a Solid Vertical Wall', International Journal of Thermal Sciences, 48(6)1161-1175.

http://dx.doi.org/:10.1016/i.ijthermalsci.2008.08.002.

Waheed,M.A.,(2009),"Heat Function Formulation of Thermal Convection in Rectangular Enclosures Filled with Porous Media", Numerical Heat Transfer -A,55, 185-204. http://dx.doi.org/ 10.1080/10407780802603246.

Wong, J. C. F. and Xie, J. L.,(2011), "Inverse Determination of a Heat Source from Natural Convection in a Porous Cavity", Computer and Fluids,52,1-14.

http://dx.doi.org/10.1016/j.compfluid.2011.07.013.

Yesiloz, G. and Aydin, O.,(2011), "Natural Convection in an Inclined Quadrantal Cavity Heated and Cooled on Adjacent Walls", Experimental Thermal and Fluid Science, 35,1169-1176. http://dx.doi.org/10.1016/j.expthermflusci.2011.04.002

Zahmatkesh, I.,(2008), "On the Importance of Thermal Boundary Conditions in Heat Transfer and Entropy Generation for Natural Convection Inside a Porous Enclosure", International Journal of Thermal Sciences, 47(3) 339-346. http://dx.doi.org/10.1016/j.ijthermalsci.2007.02.008.

Zienkiewicz, O.C and Taylor, RL,(1991),"The Finite Element Method", 4th ed. McGraw- Hill. 